\title{
From vinylsilanes to organic/inorganic hybrid materials*
}

\author{
Tien-Yau Luh \\ Department of Chemistry and Institute of Polymer Science and Engineering, \\ National Taiwan University, Taipei 106, Taiwan
}

\begin{abstract}
Alternating regioregular silylene-spaced divinylarene copolymers [(donor$\left.\mathrm{SiMe}_{2}-\right)_{n=1-3^{-}}\left(\right.$acceptor-SiMe $\left.\left.{ }_{2}\right)\right]$ are synthesized by rhodium-catalyzed hydrosilylation of bis-alkynes with bisvinylic silyl hydrides. Efficient intrachain energy transfer has been observed in these copolymers. The intensities of emission profiles depend on the relative ratio of donor-to-acceptor chromophores. The corresponding inorganic/organic hybrid materials are synthesized, and their photophysical properties are described.
\end{abstract}

Keywords: Vinylsilanes; rhodium-catalyzed hydrosilylation; energy transfer; stereoselective synthesis; light harvesting; photovoltaic applications.

\section{INTRODUCTION}

Some 15 years ago, we reported a convenient stereoselective synthesis of vinylsilanes from the corresponding benzylic dithioacetals by means of the nickel-catalyzed silylolefination reaction (eq. 1) [1,2]. The substituent(s) on the silicon atom can vary depending on the nature of the silyl-substituted methyl Grignard reagent. Accordingly, the steric environment and the chemical reactivity around the silicon atom can be tuned. For example, the introduction of an isopropoxy group on the silicon atom furnishes a useful entry for the synthesis of a range of silicon-substituted substrates for a variety of applications. This can easily be achieved by silylolefination of benzylic or allylic dithioacetals with ( $i$-PrO) $\mathrm{Me}_{2} \mathrm{SiCH}_{2} \mathrm{MgCl}$, a stable Grignard reagent having sterically bulky alkoxy substituent [3]. Thus, silyl-substituted dienes $\mathbf{1}$ has been demonstrated to be a useful intermediate for the synthesis of a variety of highly substituted cyclohexene derivatives 2 (eq. 2) [4]. Alternatively, the silicon-oxygen bond can easily be reduced to give the corresponding silyl hydride 3 [5]. Metal-catalyzed hydrosilation of bis-alkynes 4 using $\mathbf{3}$ has been demonstrated to be useful for the synthesis of silylene-spaced divinylarene copolymers 5 (eq. 3) [5,6]. This account summarizes the recent results from our laboratory on the use of such silylolefination reaction for the synthesis of a range of silicon-containing small molecules, copolymers, and hybrid materials for electroluminescent, light-harvesting, and photovoltaic applications.

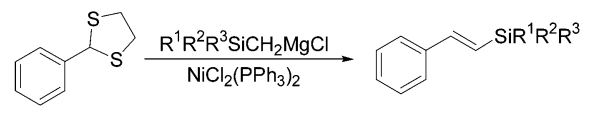

\footnotetext{
*Paper based on a presentation at the $7^{\text {th }}$ IUPAC International Conference on Heteroatom Chemistry (ICHAC-7), Shanghai, China, 21-25 August 2004. Other presentations are published in this issue, pp. 1985-2132.
} 


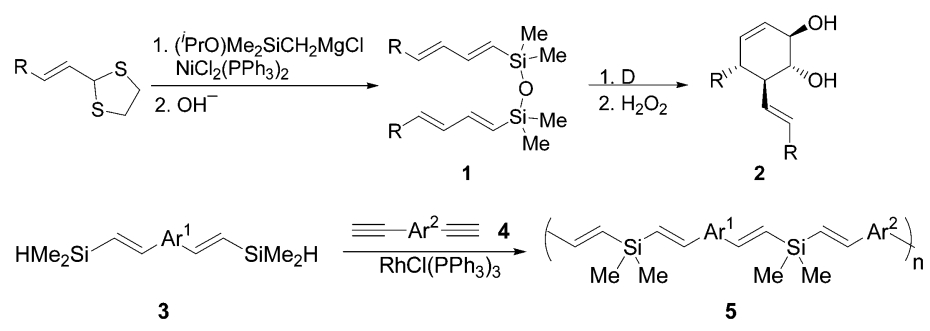

\section{VINYLSILANES FOR PROGRAMMABLE ELECTROLUMINESCENT DEVICES}

As just mentioned, the substituent(s) on the silicon atom of a vinylsilane can be easily tuned by using different kinds of the silyl-substituted Grignard reagents. Thus, the introduction of four phenyl groups in 6 would increase the steric hindrance of the molecule, which may prohibit intermolecular aggregation in the thin film [7]. Compound $\mathbf{6}$ does not exhibit strong electroluminescence but can serve as a hole-blocking material when sandwiched between the hole-transporting layer consisting of $\alpha$-NPD and the emitting layer of a thin film of $\mathrm{Alq}_{3}$. The device exhibits blue light emission due to the emission of $\alpha$-NPD layer. In the absence of $\mathbf{6}$, green emission from the $\mathrm{Alq}_{3}$ layer is observed. It is noteworthy that 6 has relatively very low $T_{\mathrm{g}}\left(30^{\circ} \mathrm{C}\right)$ which, in general, is not suitable for electroluminescent devices. As a result, after heating the device $\left(60{ }^{\circ} \mathrm{C}\right)$ or providing an electric pulse to the device, the emission color changes from blue to green. This observation suggests that the hole-blocking properties of $\mathbf{6}$ have been eliminated upon such treatment. These results give a new approach toward a programmable device. Thus, a device constituted of multilayer structure $\left(\mathrm{Alq}_{3} / \mathbf{6} / \alpha-\mathrm{NPD}\right)$ is divided into two parts. One part is heated to $60^{\circ} \mathrm{C}$, and the other is insulated with a heating sink. The section having a heating sink remains emitting in blue light, whereas the section treated by heat changes the emission color to green (Fig. 1). It is interesting to note that the performance of the device does not downgrade after such treatment. These results have brought another application by employing a very thin film of $\mathbf{6}$ between the $\mathrm{Alq}_{3}$ layer and the $\alpha$-NPD layer followed by heating or electric pulse. The functioning of these devices appears to be better than that without 6 .
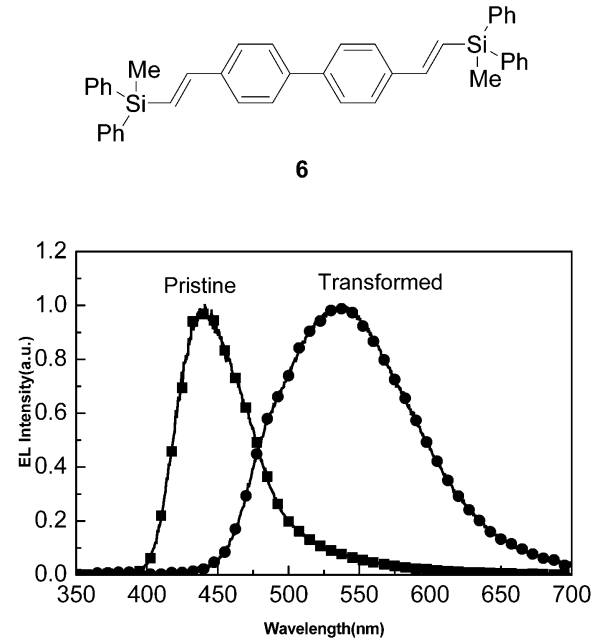

Fig. 1 Electroluminescence of the device: $\mathrm{Mg}-\mathrm{Ag} / \mathrm{Alq}_{3} / \mathbf{5} / \alpha-\mathrm{NPD} / \mathrm{TTO}$ (solid square: prestine; solid circle: after transformation at $60{ }^{\circ} \mathrm{C}$ for $3 \mathrm{~min}$ or by electric pulse $500 \mathrm{~mA} / \mathrm{cm}^{2}$ for $3 \mathrm{~s}$ ). 
As shown in Fig. 2, the brightness for the pristine device is $1000 \mathrm{~cd} / \mathrm{m}^{2}$ (blue emission), whereas that of the heat or electric pulse treated devices exhibit the brightness $10000 \mathrm{~cd} / \mathrm{m}^{2}$ (green emission) and the external efficiencies are comparable $(1 \%)$. These results suggested that the transformed devices behave as well as the pristine one. Presumably, 6 has been diffused into the $\mathrm{Alq}_{3}$ or $\alpha$-NPD layers to render the interfacial junction fuzzier [8].

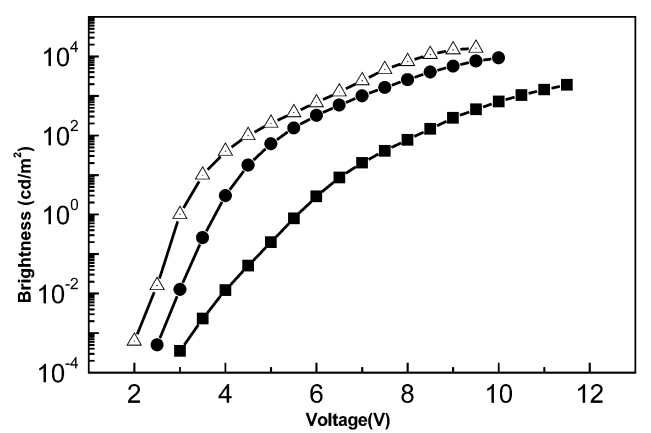

Fig. 2 Voltage-dependent brightness of the device: $\mathrm{Mg}-\mathrm{Ag} / \mathrm{Alq}_{3} / \mathbf{6} / \alpha-\mathrm{NPD} / \mathrm{ITO}$ (solid square: prestine; solid circle: after transformation at $60{ }^{\circ} \mathrm{C}$ for $3 \mathrm{~min}$ and triangle: by electric pulse $500 \mathrm{~mA} / \mathrm{cm}^{2}$ for $3 \mathrm{~s}$.

\section{LIGHT HARVESTING IN SILYLENE-SPACED COPOLYMERS}

Studies on artificial light harvesting using polymeric systems have been extensive [9]. Occasionally, moderate energy-transfer efficiencies are obtained because of aggregation which may result in quenching the fluorescence. The silylene moiety has been widely used as an insulating spacer to separate chromophores regioregularly in the polymeric backbone [5,10-13]. These silylene-spaced copolymers $\mathbf{5}$ are readily accessible by rhodium-catalyzed hydrosilylation of bis-alkynes $\mathbf{4}$ with bis-silylhydrides $\mathbf{3}$ according to eq. $3[5,10]$. Such a strategy may generate a useful synthesis of a variety of fascinating polymers that have different kinds of regioselective combination of donors and acceptors along the polymer chain. Thus, a series of alternating (donor-SiMe 2 -acceptor-SiMe ${ }_{2}$ ) copolymers has been synthesized according to eq. 3. Using chromophores $\mathbf{8}$ and $\mathbf{9}$ as a set of example, the emission profiles for a mixture of monomeric chromophores at different concentrations are compared with that of the copolymer 7. As can be seen from Fig. 3, energy transfer between the donor chromophores and the acceptor chromophores is much more efficient in the copolymer than in the mixtures of the corresponding monomeric chromophores.
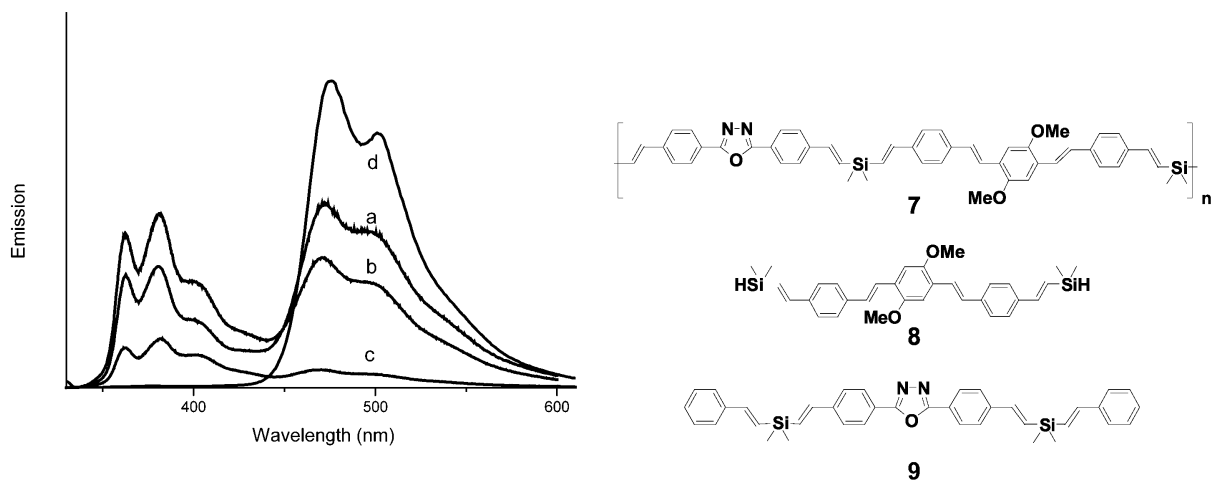

Fig. 3 Emission spectra $\left(\lambda_{\text {ex }}=324 \mathrm{~nm}\right)$ of copolymer $\mathbf{6}$ and a 1:1 mixture of monomers 7 and 8 at different concentrations $\left(\mathrm{a}=1 \times 10^{-1}, \mathrm{~b}=1 \times 10^{-2}, \mathrm{c}=1 \times 10^{-3} \mathrm{~g} / \mathrm{mL}\right)$. 
Intrachain transfer of chiroptical properties in silylene-spaced copolymers has been described [12]. Polymers 10 and $\mathbf{1 1}$ are synthesized according to eq. 3. The CD curves shown in Fig. 4a suggested that 10a and $10 \mathrm{~b}$ are mirror image. It is noteworthy that a higher concentration of the polymers is essential to observe CD spectra and aggregation of copolymers may occur at this concentration. At low concentration, the CD intensity is too weak to observe. No CD curves are observed for the corresponding monomer 12. The CD spectra for 11a and 11b are shown in Fig. 4b. The CD curves are extended to the longer wavelength region due to the absorption of the second terphenylene-tetravinylene chromophore in 11. In order to examine that the chiroptical properties are indeed transferred intramolecularly, the CD properties of a mixture of copolymers 10a and $\mathbf{1 3}$ are examined. Even at very high concentration, the $\mathrm{CD}$ profile is essentially the same as that of $\mathbf{1 0 a}$, no extension to longer wavelength being observed. No interchain transfer of chiroptical properties between 1a and $\mathbf{3}$ would occur under these conditions.
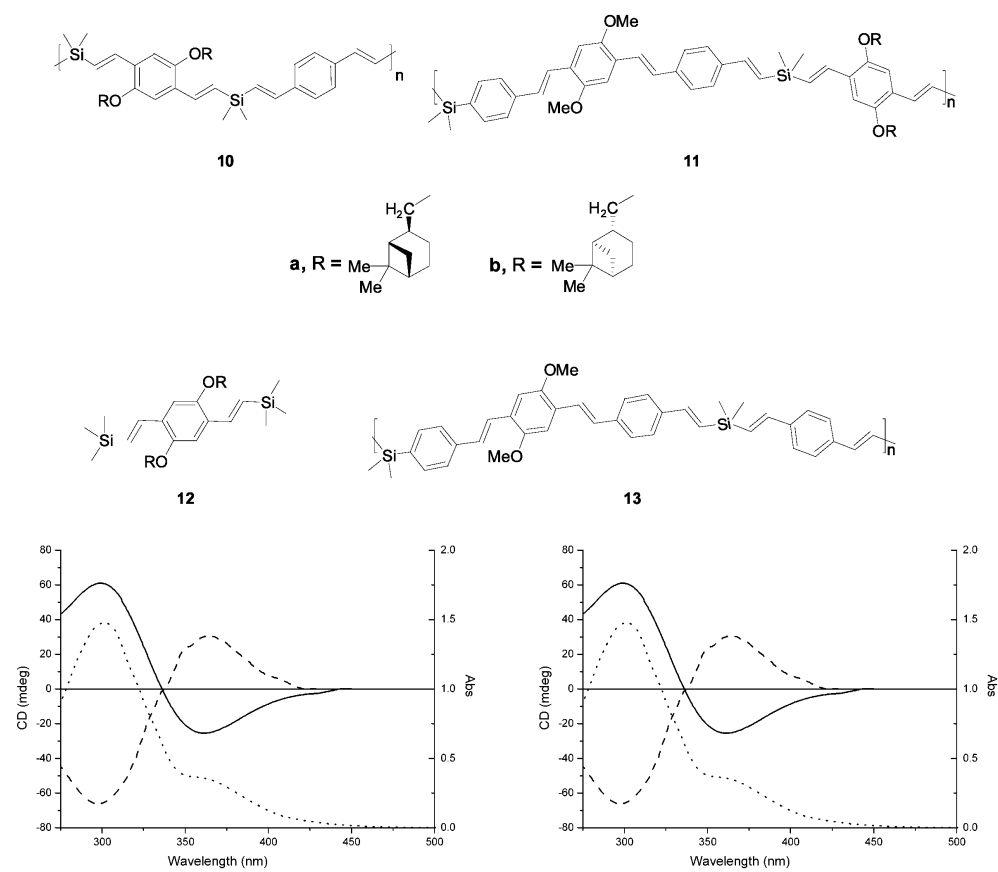

Fig. 4 (a) CD curves of 10a (solid line) and 10b (dashed line) (1.5 g/L in chloroform), and the UV-vis spectrum of 10a (dotted line). (b) CD curves of 11a (solid line) and 11b (dashed line) (1 g/L in chloroform), and the UV-vis spectrum of 11a (dotted line).

The $\left[(\text { donor-SiMe })_{n}\right.$-acceptor-SiMe $\left.{ }_{2}\right]$ copolymers 14-16 (where $n=1-3$ have been synthesized from the corresponding bis-alkynes $\mathbf{1 7}$ and $\mathbf{1 8}$ (eqs. 4 and 5) according to eq. 3 . The fluorescence spectra of copolymers 14-16 ( $\lambda_{\mathrm{ex}}=300 \mathrm{~nm}$, the absorption maximum for the donor chromophore) are shown in Fig. 5. It is clear that the intensity of the emission profiles increases with increasing ratio of the donor chromophore against the acceptor chromophore [12]. The efficiencies of energy transfer for $\mathbf{1 4}$ and $\mathbf{1 5}$ are nearly quantitative, and that of $\mathbf{1 6}$ is over $90 \%$. It is noteworthy that the distances between the donor chromophores and the acceptor chromophore in $\mathbf{1 4}$ and $\mathbf{1 5}$ are the same, whereas those in $\mathbf{1 6}$ are different. Such discrepancy may result in different efficiencies in energy transfer in these polymers. Upon excitation at $360 \mathrm{~nm}$ ( $\lambda_{\max }$ for the acceptor chromophore), the emission spectrum of 16 is also shown in Fig. 3 for comparison. The intensity of this latter emission is apparently lower than that excited at $300 \mathrm{~nm}$. These results clearly indicate that the light harvesting plays an important role to enhance the emission intensity [13]. 


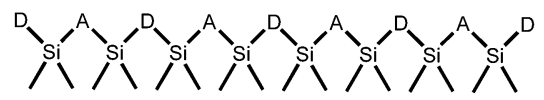

14

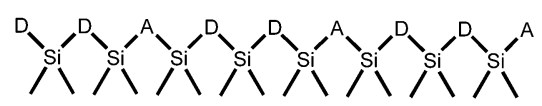

15
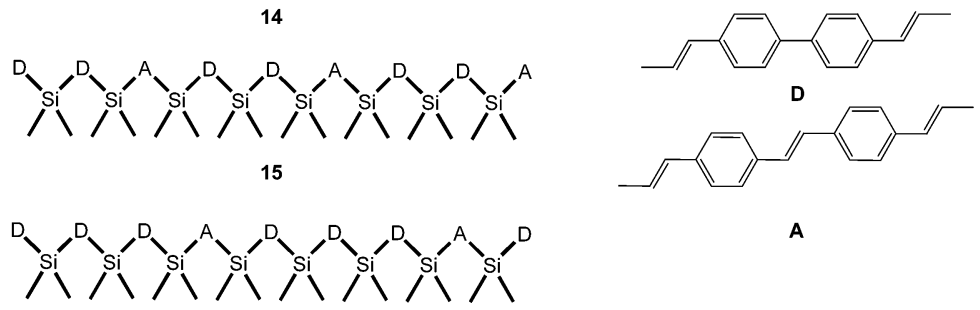

16
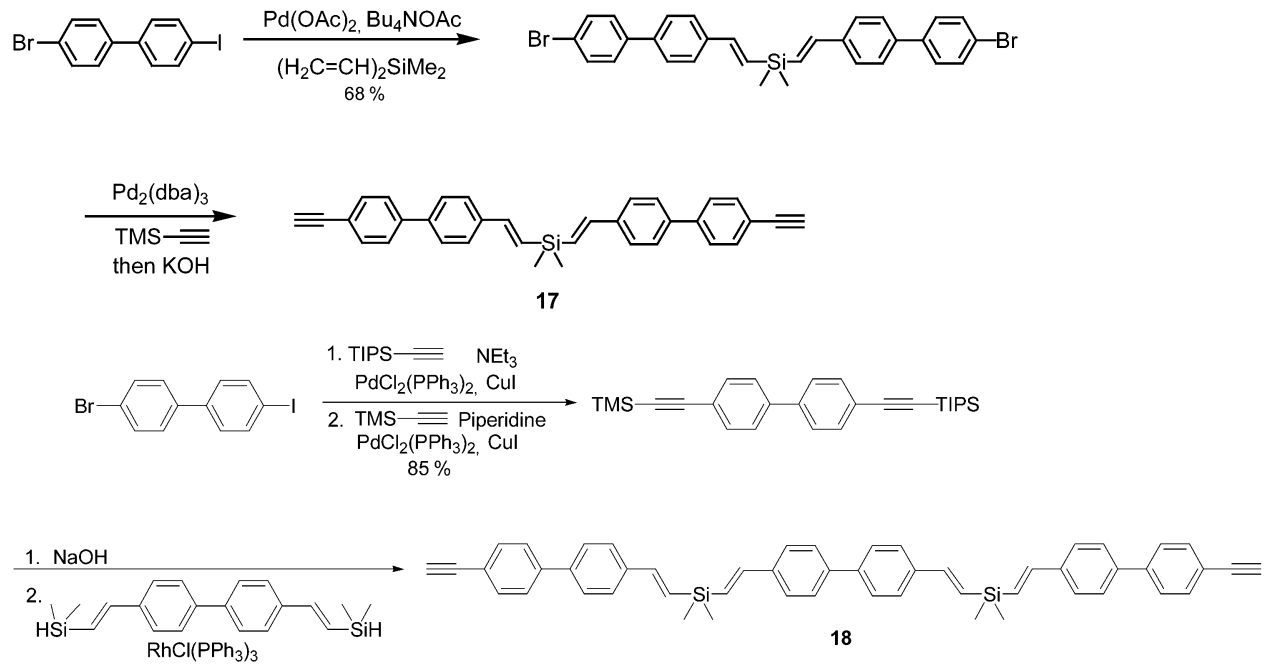

3. TBAF

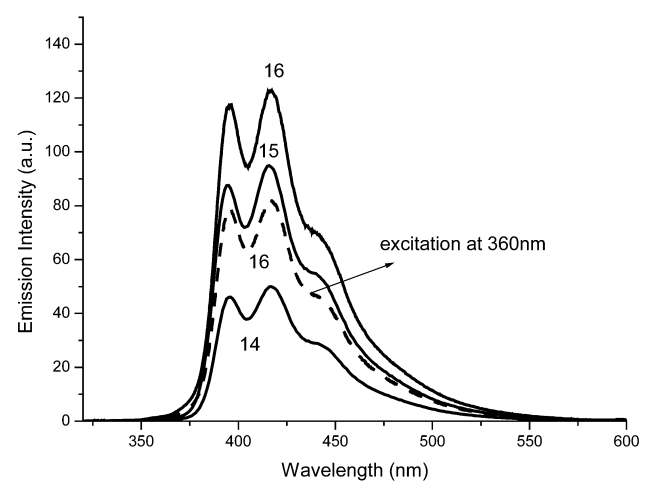

Fig. 5 Emission spectra of 14-16 $\left(\lambda_{\mathrm{ex}}=300 \mathrm{~nm}\right)$.

\section{LIGHT HARVESTING IN SILICON-CONTAINING ORGANIC/INORGANIC HYBRID MATERIALS}

As described in the previous section, efficient intrachain energy transfer between chromophores can occur in silylene-spaced divinylarene copolymers. It is envisaged that incorporation of such chromophores covalently bonded to an inorganic silica matrix to disperse the donor and acceptor species may 
also facilitate inter-chromophore energy transfer. Since the vinylsilane moiety (e.g., 19) can easily be generated by the olefination of benzylic dithioacetals (eqs. 1 and 2) [1,2], attempts to polymerize 19 under various conditions are unsuccessful. Intriguingly, dimeric cyclophanes $\mathbf{2 0}$ are obtained in moderate to good yield (eq. 6) [14]. Presumably, the isopropoxy group is less reactive and the reaction becomes more selective to give $\mathbf{2 0}$.

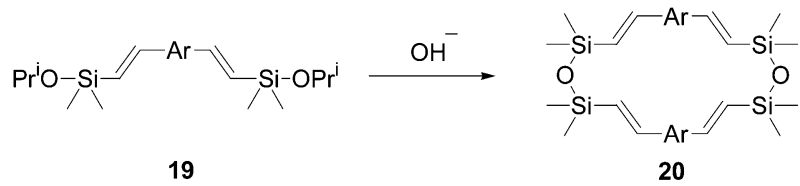

Replacement of the isopropoxy group in $\mathbf{1 9}$ into a more reactive and less hindered alkoxy group is desirable. The synthesis involves a conversion of isopropoxysilanes $\mathbf{1 5}$ into the more reactive ethoxysilanes 16 via silyl hydrides (eq. 6) [13]. It is interesting to note that the substrates contain double bond and palladium is an active catalyst for hydrogenation of double bonds. Under the reaction conditions, one mole of hydrogen is generated, which can add to the double bond. In order to avoid this side reaction, addition of norbornadiene can efficiently trap the hydrogen generated and ethoxyslianes thus obtained are used for the preparation of hybrid materials 17 (eq. 7) [14].

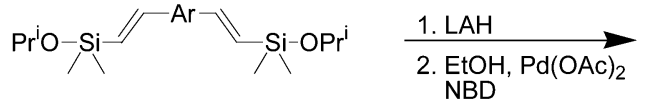

19

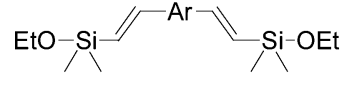

21

Bisethoxysilanes 21 readily form organic/inorganic hybrid materials upon treatment with tetraethoxysilane in the presence of $\mathrm{Bu}_{4} \mathrm{NF}$. Accordingly, hybrid materials $\mathbf{2 2}$ containing more than one kind of chromophores can be obtained by this strategy. A representative cartoon is shown in eq. 8 . The ratio of donor and acceptor chromophores can be tuned, and the emission profiles are shown in Fig. 6.
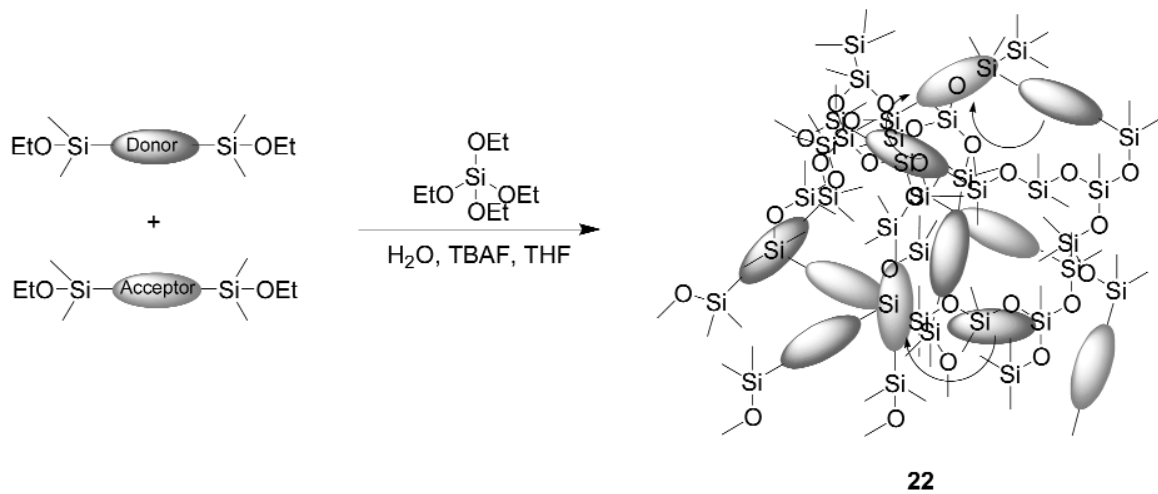

As can be seen from Fig. 6, the emission profile 22 having $100 \%$ donor chromophore exhibits structureless spectrum, presumably due to aggregation. Similar aggregation profiles are observed when the mole fraction of the acceptor chromophore exceeds $80 \%$. Efficient energy transfer apparently occurs in these hybrid materials having different ratios of donor and acceptor chromophores ranging from 80:20 to 40:60. No excimer-like emission is observed in these mixed hybrid materials. It seems likely that the acceptor chromophores are segregated nicely and the donor and acceptor chromophores are distributed homogeneously in this hybrid system. Preliminary examination also shows that the energy transfer in these hybrid materials also occur quite efficiently (>80\%). 


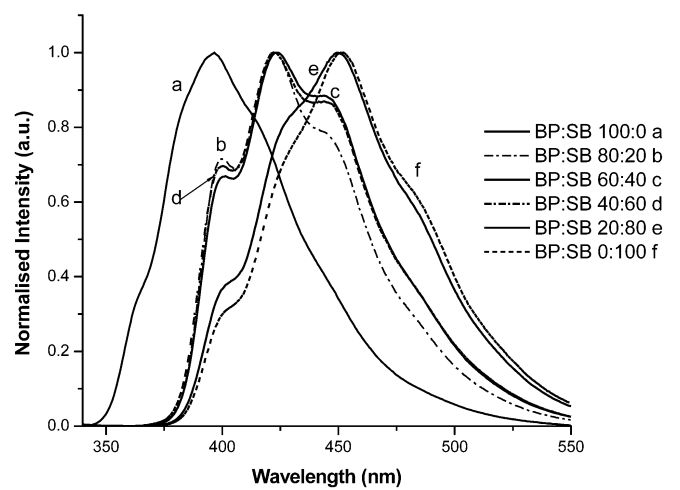

Fig. 6 Emission profiles of $\mathbf{1 7}$ having different ratios of donor and acceptor chromophores.

\section{CONCLUSION}

We have demonstrated a convenient synthesis of a range of silicon-containing conjugated chromophores from the corresponding benzylic dithioacetals. The silicon moieties have been shown to serve as a steric bulky substituent in small molecules, an insulating spacer in polymers, and a three-dimensional framework in hybrid materials. These materials have been demonstrated to exhibit unique optoelectronic properties and can be used in electroluminescence, light-harvesting models. The potential use of these materials for photovoltaic and other applications is in progress.

\section{ACKNOWLEDGMENTS}

The author wishes to thank his coworkers, whose names are shown in the references, for their valuable contributions to this work. Thanks are also due to the National Science Council and the Ministry of Education of the Republic of China for support.

\section{REFERENCES}

1. (a) Z.-J. Ni and T.-Y. Luh. J. Org. Chem. 53, 2129, 5582 (1988); (b) Z.-J. Ni, P.-F. Yang, D. K. P. Ng, Y.-L. Tzeng, T.-Y. Luh. J. Am. Chem. Soc. 112, 9356 (1990); (c) Z.-J. Ni and T.-Y. Luh. Org. Synth. 70, 240 (1991).

2. For reviews, see: T.-Y. Luh. Acc. Chem. Res. 24, 257 (1991); Pure Appl. Chem. 68, 105 (1996).

3. K. Tamao, N. Ishida, Y. Ito, M. Kumada. Org. Synth. 69, 96 (1990).

4. R.-M. Chen, W.-W. Weng, T.-Y. Luh. J. Org. Chem. 60, 3272 (1995).

5. (a) R.-M. Chen, K.-M. Chien, K.-T. Wong, B.-Y. Jin, T.-Y. Luh, J.-H. Hsu, W. Fann. J. Am. Chem. Soc. 119, 11321 (1997); (b) R.-M. Chen and T.-Y. Luh. Tetrahedron 54, 1197 (1998).

6. For a review, see: T.-Y. Luh, R.-M. Chen, T.-Y. Hwu, S. Basu, C.-W. Shiau, W.-Y. Lin, B.-Y. Jin, C. C. Hsu. Pure Appl. Chem. 73, 243-246 (2001).

7. C. C. Wu, C. W. Chen, Y. T. Lin, H.-L. Yu, J.-H. Hsu, T.-Y. Luh. Appl. Phys. Lett. 79, 3023 (2001).

8. C.-W. Chen, T.-Y. Cho, C. C. Wu, H.-L. Yu, T.-Y. Luh. Appl. Phys. Lett. 81, 1570 (2002).

9. (a) M. A. Fox. Acc. Chem. Res. 25, 569 (1992); (b) S. E. Webber. Chem. Rev. 90, 1469 (1990); (c) M. Nowakowska, P. V. Foyle, J. E. Guillet. J. Am. Chem. Soc. 115, 5975 (1993); (d) K. Hisada, S. Ito, M. Yamamoto. Langmuir 11, 996 (1995); (e) X. Schultze, J. Serin, A. Adronov, J. M. J. Fréchet. Chem. Commun. 1160 (2001); (f) D. M. Russel, C. A. Arias, R. H. Friend, C. Silvia, C. Ego, A. C. Grimsdale, K. Müllen. Appl. Phys. Lett. 80, 2204 (2002).

10. Y.-J. Cheng, T.-Y. Hwu, J.-H. Hsu, T.-Y. Luh. Chem. Commun. 2336 (2002). 
11. T.-Y. Hwu, S. Basu, R.-M. Chen, Y.-J. Cheng, J.-H. Hsu, T.-Y. Luh. J. Polym. Sci., Part A: Polym. Chem. 41, 2218 (2002).

12. Y.-J. Cheng, H. Liang, T.-Y. Luh. Macromolecules 36, 5921 (2003).

13. Y.-J. Cheng and T.-Y. Luh. Chem. Eur. J. 10, 5361 (2004).

14. S. Sudhakar and T.-Y. Luh. J. Org. Chem. 67, 6860 (2002).

15. S. Sudhakar, G. H. Lee, Y. Wang, J.-H. Hsu, T.-Y. Luh. J. Organomet. Chem. 646, 167 (2002).

16. S. Sudhakar and T.-Y. Luh. J. Org. Chem. 67, 6860 (2002).

17. S. Sudhakar, S. Sudhakar, C.-H. Chen, C.-L. Lin, M.-Y. Yeh, K.-Y. Liu, T.-Y. Luh. Unpublished results. 